\title{
Measurement of hadronic cross sections at CMD-3
}

\author{
V.L. Ivanov ${ }^{* a, b}$, on behalf the CMD-3 collaboration \\ ${ }^{a}$ Budker Institute of Nuclear Physics, SB RAS, Novosibirsk, 630090, Russia \\ ${ }^{b}$ Novosibirsk State University, Novosibirsk, 630090, Russia \\ E-mail: V.L.Ivanov@inp.nsk.su
}

\begin{abstract}
This paper reports a current status of the measurement of the hadronic cross sections in the c.m. energy range from 0.32 to $2.0 \mathrm{GeV}$ with the CMD-3 detector at the VEPP-2000 electron-positron collider. The overall size of the data, acquired by the CMD-3 in the runs of 2010-2013 and 20172019 years, is about $250 \mathrm{pb}^{-1}$. The recent results of data analysis for various exclusive modes of $e^{+} e^{-} \rightarrow$ hadrons are described.
\end{abstract}

European Physical Society Conference on High Energy Physics - EPS-HEP2019 -

10-17 July, 2019

Ghent, Belgium

${ }^{*}$ Speaker. 


\section{Introduction}

The CMD-3 detector [1] at the VEPP-2000 collider [2] in Novosibirsk carries out a comprehensive study of the exclusive cross-sections of $e^{+} e^{-} \rightarrow$ hadrons in the c.m. energy range from 0.32 up to $2 \mathrm{GeV}$. The CMD-3 results provide an important input for calculation of the hadronic contribution to the muon anomalous magnetic moment (AMM). Currently there are world-wide efforts to improve the accuracy of this calculation to match the expected precision of the Fermilab measurement [3], which started data taking in 2018. The best precision is still achieved by integrating the measured total cross-section of $e^{+} e^{-} \rightarrow$ hadrons. The VEPP-2000 energy range gives the major hadronic contribution to AMM, both to the hadronic vacuum polarization itself ( $\sim 92 \%)$ and to its uncertainty [4]. Other interesting topics of the CMD-3 physics program include a study of hadron cross-sections at the nucleon-antinucleon threshold and a search for two-photon production of $\mathrm{C}$-even resonances.

The first round of data taking with CMD-3 in the whole available energy range was done in 2010-2013. After a three-year break for collider and detector upgrades, data taking resumed in 2017-2019 with a 2-3 times higher luminosity. Overall, about $250 \mathrm{pb}^{-1}$ have been collected so far. In this report we present the overview of the results of data analysis, including various modes of electron-positron annihilation with up to six pions or two kaons and pions in the final state.

\section{CMD-3 Detector}

The coordinates, angles and momenta of charged particles in CMD-3 are measured by the cylindrical drift chamber (DC). The cylindrical multiwire double layer proportional Z-chamber (ZC) provides z-coordinate determination with accuracy $\sim 0.5 \mathrm{~mm}$. The endcap calorimeter consists of $640 \mathrm{BGO}$ crystals with a thickness $13.4 \mathrm{X}_{0}$. The barrel calorimeter is placed outside of the thin $0.08 \mathrm{X}_{0}$ superconducting solenoid with $1.3 \mathrm{~T}$ magnetic field and consists of two subsystems: inner Liquid Xenon (LXe) ionization calorimeter $\left(5.4 \mathrm{X}_{0}\right)$ and outer CsI scintillation calorimeter $\left(8.1 \mathrm{X}_{0}\right)$. The photon conversion point in the LXe is measured with $\sim 1 \div 2 \mathrm{~mm}$ precision. The muon range system is located outside of the magnetic yoke and consists of 36 scintillation counters which have time resolution about $1 \mathrm{~ns}$. More detailed detector description can be found in [1].

\section{Data Set and Luminosity Measurement}

Currently the data set, collected by the CMD-3, includes $\sim 65 \mathrm{pb}^{-1}$ at $\sqrt{s}<1.0 \mathrm{GeV}$ and $\sim 185 \mathrm{pb}^{-1}$ at $\sqrt{s}>1.0 \mathrm{GeV}$, including $14 \mathrm{pb}^{-1}$ of data at nucleon-antinucleon production threshold region. During the experiment the beam energy was determined by measuring the momenta of Bhabha events in DC with accuracy $\sim 1 \div 3 \mathrm{MeV}$ and, starting from 2013, using the Compton backscattering technique with accuracy $\sim 50 \mathrm{keV}$ [5]. The peak collider luminosity was $\sim 4$. $10^{31} \mathrm{~cm}^{-2} \mathrm{~s}^{-1}$. The integral luminosity was determined using the events of Bhabha scattering, and, for cross-check, the $e^{+} e^{-} \rightarrow \gamma \gamma$ events. The systematic uncertainty of the luminosity measurement at high energies is now estimated as $1 \%$ [6]. 


\section{Pion Form Factor Measurement}

One of the main goals of the CMD-3 experiment is to reduce a systematic uncertainty of the cross section of two-pion production to the level smaller than $0.5 \%$, which corresponds to $\sim 0.35 \mathrm{ppm}$ uncertainty in the AMM. The data sample, collected by CMD-3 in 2011-2018 at $\sqrt{s}<1.0 \mathrm{GeV}$ is $\sim 3$ times larger than that of BaBar, KLOE and BES. To control the systematic uncertainty, the $\pi^{+} \pi^{-}$events selection is performed using two independet methods - using particles momenta or their energy deposition in the calorimeter. In both cases 2-dimensional binned likelyhood function maximization is performed to obtain the ratio of numbers of $\pi^{+} \pi^{-}$and $e^{+} e^{-}$ events. Currently the systematic uncertainty for pion form factor measurement is estimated to be 0.4-0.9\% (momentum-based approach) and 1.5\% (energy-based approach), the preliminary results are published in [7].

\section{Study of the Processes $e^{+} e^{-} \rightarrow \pi^{+} \pi^{-} 2 \pi^{0}, 2 \pi^{+} 2 \pi^{-}$}

Using the large data sample of $e^{+} e^{-} \rightarrow \pi^{+} \pi^{-} 2 \pi^{0}$ (64k events) and $e^{+} e^{-} \rightarrow 2 \pi^{+} 2 \pi^{-}$(72k events) collected by the CMD-3 the simultaneous analysis of these two final states was performed.

Due to the limited detector accentance the detection efficiency strongly depends on the production dynamics, which involves the mechanisms $\omega(782) \pi^{0} \rightarrow \rho(770) 2 \pi^{0}, a_{1}^{ \pm} \pi \rightarrow \rho^{ \pm}(770) \pi$, $a_{1}^{ \pm} \pi^{\mp} \rightarrow \sigma \pi^{+} \pi^{-}, h_{1} \pi^{0} \rightarrow \rho(770) 2 \pi^{0}, \rho^{+}(770) \rho^{-}(770), \rho(770) f_{0}$ and others. To find the amplitudes of these mechanismcs the unbinned fit of both $\pi^{+} \pi^{-} 2 \pi^{0}$ and $2 \pi^{+} 2 \pi^{-}$final states was done at each c.m. energy point. The contribution of each mechanism to the total hadronic current of the process was calculated using the effective lagrangian approach.

The performed unbinned fit results in a good data/Monte Carlo agreement for both final states, see Figs. 1-2. Using the amplitudes obtained in the unbinned fit the contribution of each mechanism to the total cross section can be calculated (neglecting the interference), see Fig. 3. The more detalies about this analysis can be found in [8].
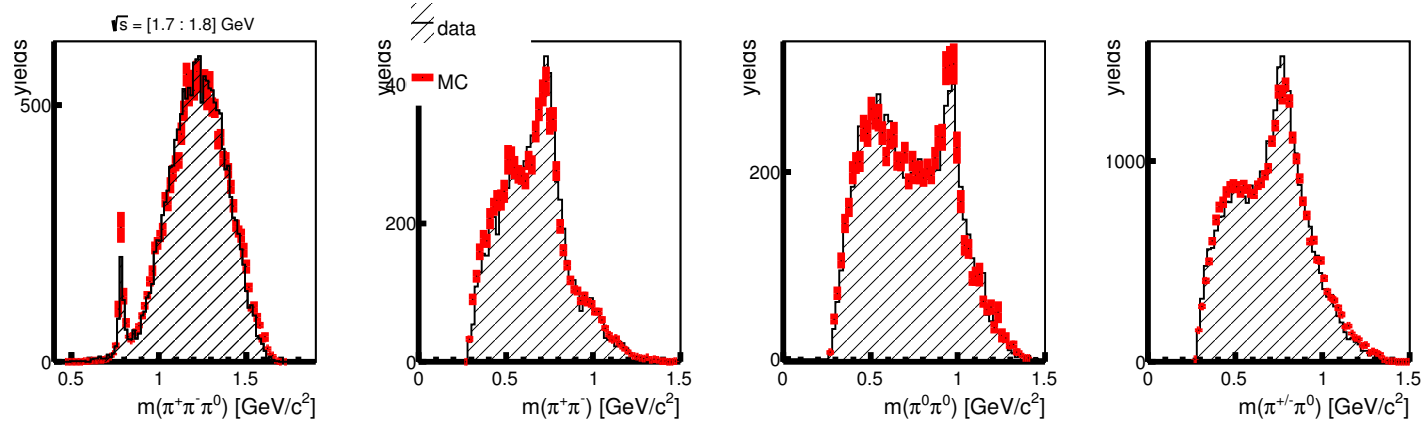

Figure 1: The data-MC comparison of the $\pi^{+} \pi^{-} \pi^{0}, \pi^{+} \pi^{-}, \pi^{0} \pi^{0}$ and $\pi^{ \pm} \pi^{0}$ mass spectra for the process $\mathrm{e}^{+} \mathrm{e}^{-} \rightarrow 2 \pi^{0} \pi^{+} \pi^{-}$in the energy range $\sqrt{s}=1.7 \div 1.8 \mathrm{GeV}$.

\section{Study of the Processes $e^{+} e^{-} \rightarrow K^{+} K^{-} \eta$}

Since the intermediate mechanism $e^{+} e^{-} \rightarrow \phi(1680) \rightarrow \phi(1020) \eta$ dominates in this process, the study of the latter provides a good opportunity for the $\phi(1680)$ meson parameters measurement. 

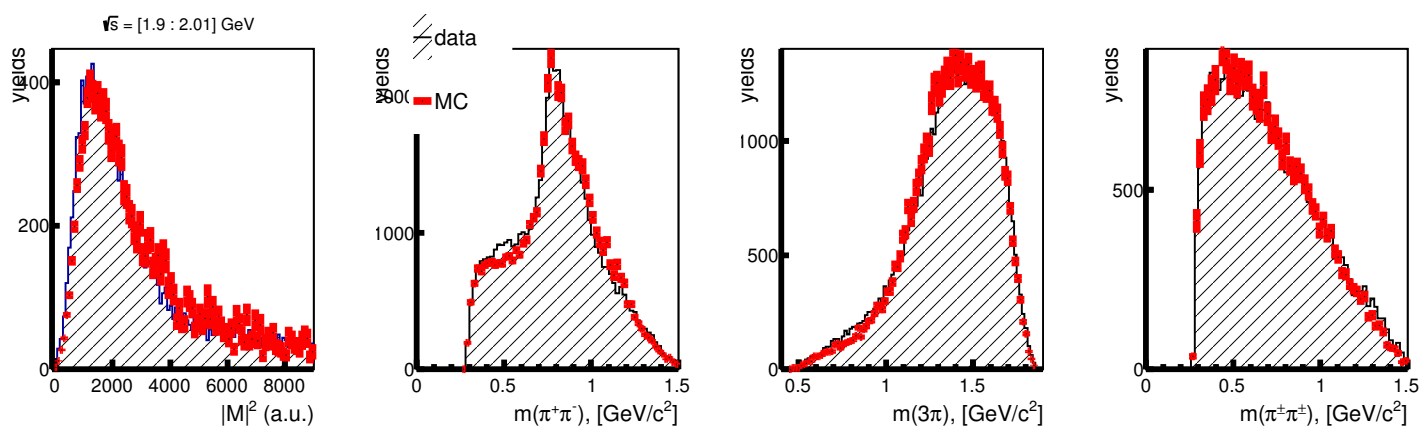

Figure 2: The data-MC comparison of the squared matrix element $|M|^{2}$ and $\pi^{+} \pi^{-}, 2 \pi^{ \pm} \pi^{\mp}$ and $\pi^{ \pm} \pi^{ \pm}$mass spectra for the process $\mathrm{e}^{+} \mathrm{e}^{-} \rightarrow 2 \pi^{+} 2 \pi^{-}$in the energy range $\sqrt{s}=1.9 \div 2.01 \mathrm{GeV}$.

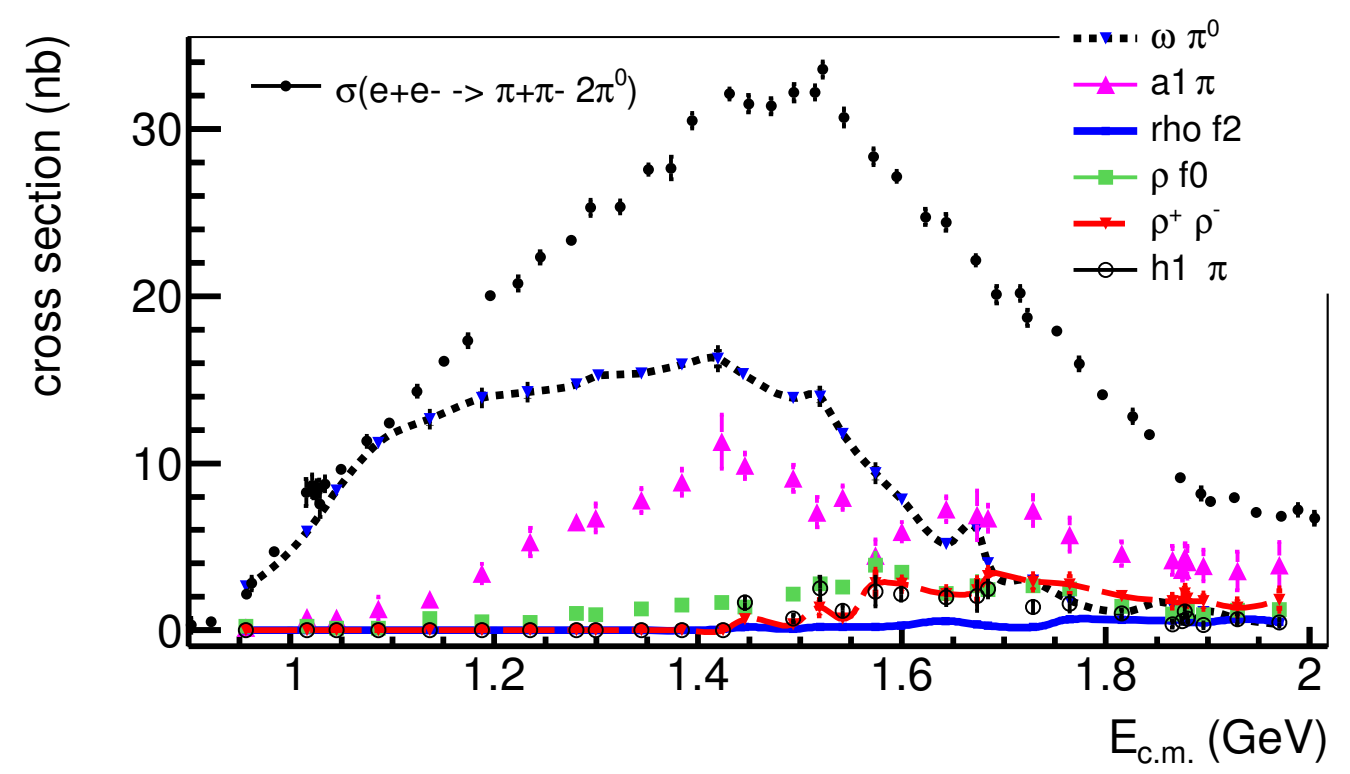

Figure 3: Cross sections calculated for different components of the matrix element and the total $\mathrm{e}^{+} \mathrm{e}^{-} \rightarrow$ $2 \pi^{0} \pi^{+} \pi^{-}$cross section (black circles).

In our analysis we consider $\eta$ meson as a recoil particle, thus all decay modes of $\eta$ are used. The kaon identification (kaon/pion separation) is based on the $d E / d x$ of the tracks and uses likelyhood function maximization. The total number of selected signal events is $3009 \pm 67$. The results for the $e^{+} e^{-} \rightarrow \phi(1020) \eta$ process cross section, based on the data of 2011-2012 and 2017 runs, are shown in Fig. 4 along with Babar data. The final results including the $\phi(1680)$ meson parameters determination are published in [9].

\section{Study of the Process $e^{+} e^{-} \rightarrow \eta \pi^{+} \pi^{-}$}

Our study of this process uses the $\eta \rightarrow \gamma \gamma$ decay mode. The dominant mechanism in this processs was found to be the $\rho(770) \eta$. The number of signal events is obtained from the fitting 
of the two photon invariant mass distibution near $\eta$ meson mass. The total number of the selected signal events in the c.m. energy range from 1.2 to $2.0 \mathrm{GeV}$ is $13426 \pm 206$. The preliminary results for the Born cross section are shown in Fig. 5. The cross section shape is fitted with the contribution of $\rho(770), \rho(1450$ and $\rho(1700)$ resonances. The more details about this analysis can be found in [10].

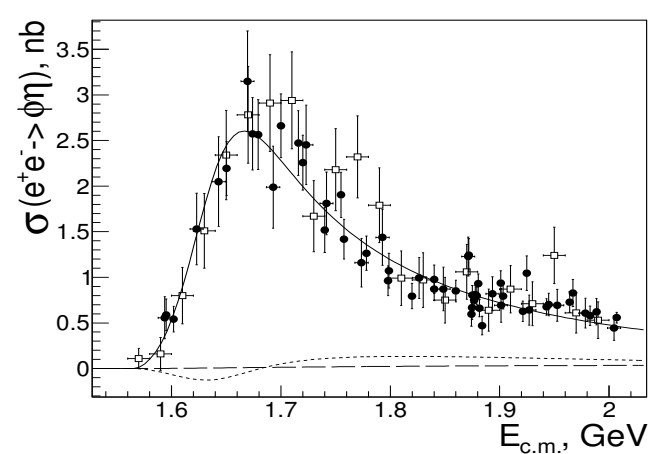

Figure 4: Cross section of $e^{+} e^{-} \rightarrow \phi(1020) \eta$ process, measured by the CMD-3 (red), and by the BaBar (black).

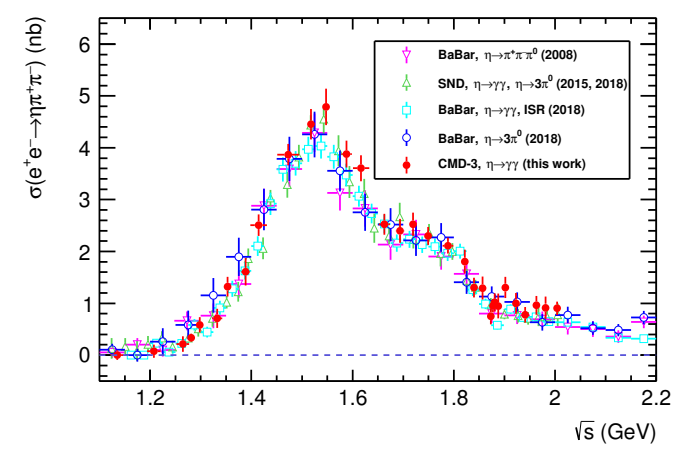

Figure 5: Born cross section for $e^{+} e^{-} \rightarrow \eta \pi^{+} \pi^{-}$ process, measured at the CMD-3, SND and BaBar.

\section{Study of the Processes $e^{+} e^{-} \rightarrow 3 \pi^{+} 3 \pi^{-} \pi^{0}$}

On the basis of $56.7 \mathrm{pb}^{-1}$ we performed the fist measurement of the total cross section of $e^{+} e^{-} \rightarrow 3 \pi^{+} 3 \pi^{-} \pi^{0}$ process in the c.m. energy range from 1.6 to $2.0 \mathrm{GeV}$, see Fig. 6 . The total cross section was described as a contribution of the reactions $e^{+} e^{-} \rightarrow 2 \pi^{+} 2 \pi^{-} \eta$ and $e^{+} e^{-} \rightarrow 2 \pi^{+} 2 \pi^{-} \omega(782)$. The final results are published in [11].

\section{Searching for $e^{+} e^{-} \rightarrow D^{* 0}(2007)$}

The process $e^{+} e^{-} \rightarrow D^{* 0}(2007)$ can be used to probe the Beyond Standard Model physics. The estimation of the lower limit on the branching fraction $B\left(D^{* 0}(2007) \rightarrow e^{+} e^{-}\right)$in the Standard Model gives $(0.1-5) \times 10^{-19}$ - a much smaller value than in some BSM models. For instance, in the model with $Z^{\prime}$-mediated gauge interaction the branching fraction is $B\left(e^{+} e^{-} \rightarrow D^{* 0}(2007)\right)<$ $2.5 \times 10^{-11}[12]$.

We performed the search for the events of $e^{+} e^{-} \rightarrow D^{* 0}(2007)$ process in the $D^{* 0}(2007) \rightarrow$ $D^{0} \pi^{0}\left(B\left(D^{* 0} \rightarrow D^{0} \pi^{0}\right) \approx 64.6 \pm 0.9 \%\right)$ and $D^{* 0}(2007) \rightarrow D^{0} \gamma\left(B\left(D^{* 0} \rightarrow D^{0} \gamma\right) \approx 35.3 \pm 0.9 \%\right)$ decay modes. The $D^{0}$ was searched in the $K^{+} \pi^{+} 2 \pi^{-}$decay mode $\left(B\left(D^{0} \rightarrow K^{+} \pi^{+} 2 \pi^{-}\right) \approx 8.11 \pm\right.$ $0.15 \%)$.

The search was based on the $3.7 \mathrm{pb}^{-1}$ of integrated luminosity, collected at the c.m. energy point $2006.632 \pm 0.008 \mathrm{MeV}$ and the beam energy spread was $0.954 \pm 0.053 \mathrm{MeV}$. We got 2 candidates for $D^{0} \gamma$ events with the estimated background of $1.2 \pm 0.5$ and 1 candidate for $D^{0} \pi^{0}$ with $1.5 \pm 0.7$ background. The resulting upper limit, estimated using the Bayesian Approach, is $B\left(D^{* 0}(2007)<1.6 \times 10^{-6}\right)$ at $90 \%$ C.L. See more details on this analysis in [13]. 


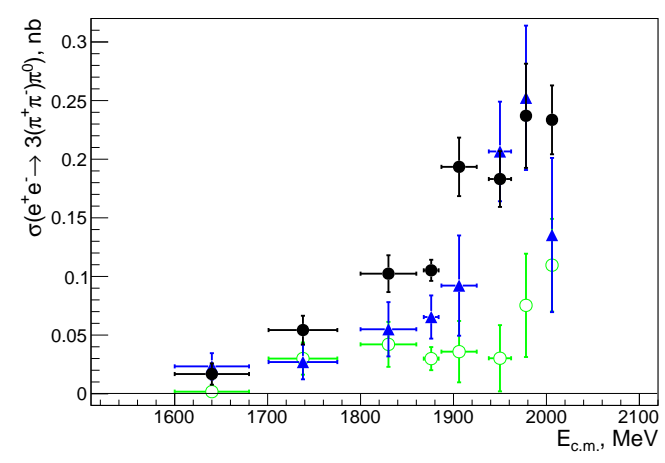

Figure 6: The $e^{+} e^{-} \rightarrow 3\left(\pi^{+} \pi^{-}\right) \pi^{0}$ cross section measured with the CMD-3 detector at VEPP-2000 (dots). The contribution from the $e^{+} e^{-} \rightarrow 2\left(\pi^{+} \pi^{-}\right) \eta$ and $e^{+} e^{-} \rightarrow 2\left(\pi^{+} \pi^{-}\right) \omega$ reactions are shown by triangles and open circles, respectively.

\section{Multihadron production at nucleon-antinucleon threshold}

During the experimental runs of 2017 we performed the detailed scan of nucleon-antinucleon threshold region with the step of $\sim 0.8 \mathrm{MeV}$. We confirmed the existence of the dip structures in the $e^{+} e^{-} \rightarrow 3 \pi^{+} 3 \pi^{-}$and $e^{+} e^{-} \rightarrow K^{+} K^{-} \pi^{+} \pi^{-}$cross section, see Figs. $7-8$. The behaviour of the cross sectionc near threshold is reasonably described by the Milstein-Salnikov parametrization, see [14]. Hovewer, we see no dip structures in the cross sections of $e^{+} e^{-} \rightarrow 2 \pi^{+} 2 \pi^{-}$and $e^{+} e^{-} \rightarrow \pi^{+} \pi^{-} 4 \pi^{0}$. For more detailes about this study see [15].

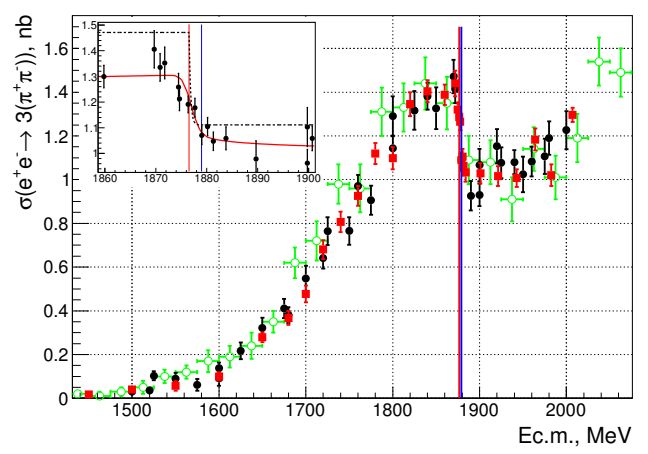

Figure 7: The $e^{+} e^{-} \rightarrow 3\left(\pi^{+} \pi^{-}\right)$cross section, measured by the CMD-3 in the 2017 runs (red), in 2011-2012 (black), and by BaBar (green). The inset shows the visible cross section with the fit. The lines show nucleon-antinucleon thresholds.

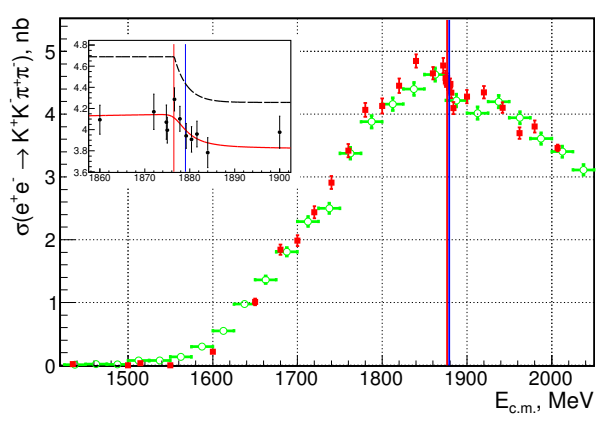

Figure 8: The $e^{+} e^{-} \rightarrow K^{+} K^{-} \pi^{+} \pi^{-}$cross section, measured by the CMD-3 in the 2017 runs (red), and by BaBar (green). The inset shows the visible cross section with the fit. The lines show nucleon-antinucleon thresholds.

\section{Summary and Conclusion}

The VEPP-2000 $e^{+} e^{-}$collider, CMD-3 and SND detectors successfully operate to collect $\sim 1 \mathrm{fb}^{-1}$ of data in the next 5-10 years. The current integrated luminosity is about $250 \mathrm{pb}^{-1}$ for 
each detector, which is enough to provide the results with comparable or better precision with respect to previous experiments. Data analysis for many hadronic final states is in progress.

\section{Acknowledgements}

The authors are grateful to the VEPP-2000 team for excellent machine operation.

This work is partially supported by the Russian Foundation for Basic Research grants RFBR 17-02-00897 (in part, related to Section 6), 17-02-00327-a (in part, related to Section 5).

\section{References}

[1] B.I.Khazin et al., Nucl. Phys .B, Proc. Suppl. 376181 (2008).

[2] I.A.Koop et al., Nucl. Phys. B, Proc.Suppl. 371263 (2008).

[3] A.Chapelain et. al., EPJ Web Conf. 13708001 (2017).

[4] M.Davier et al., EPJ C 31503 (2003).

[5] E.V.Abakumova et al., Phys. Rev. Lett. 110140402 (2013).

[6] A.E.Ryzhenenkov et al., EPJ Web Conf. 21204011 (2019).

[7] F.V.Ignatov et al., EPJ Web Conf. 21802006 (2019).

[8] E.A.Kozyrev et.al EPJ Web Conf., 21203008 (2019).

[9] V.L.Ivanov et al. Phys. Lett. B 798134946 (2019).

[10] S.S.Gribanov et al. arXiv:1907.08002 [hep-ex].

[11] R.R.Akhmetshin et al. Phys. Lett. B 792 419-423 (2019).

[12] A.Khodjamirian et al. JHEP 1511, 142 (2015).

[13] D.N.Shemyakin et al. EPJ Web Conf. 212, 02011 (2019).

[14] A.I.Milstein and S.G.Salnikov, Nuclear Phys. A 977 60-68 (2018).

[15] R.R.Akhmetshin et al. Phys. Lett. B 794 64-68 (2019). 